\title{
Analysis of Using Resources in Business Process Modeling and Simulation
}

\author{
Olegas Vasilecas ${ }^{1,2}$, Evaldas Laureckas ${ }^{3}$, Audrius Rima $^{4}$ \\ ${ }^{1,4}$ Vilnius Gediminas Technical University, Lithuania, ${ }^{2,3}$ Klaipeda University, Lithuania
}

\begin{abstract}
One of the key purposes of Business Process Model and Notation (BPMN) is to support graphical representation of the process model. However, such models have a lack of support for the graphical representation of resources, whose processes are used during simulation or execution of process instance. The paper analyzes different methods and their extensions for resource modeling. Further, this article presents a selected set of resource properties that are relevant for resource modeling. The paper proposes an approach that explains how to use the selected set of resource properties for extension of process modeling using BPMN and simulation tools. They are based on BPMN, where business process instances use resources in a concurrency manner.
\end{abstract}

Keywords - Business Process Model and Notation (BPMN), business process models, resources, resources models, resources modeling, resource aspect modeling.

\section{INTRODUCTION}

The aim of BPMN is to support easily understandable models, define and graphically represent business processes [2], [8], [16] and [22]. BPMN represents business process control logic and workflow [21]. However, in order to simulate the BPMN model, first, it has to encounter various restrictions of this notation. Usually, restrictions are concerned with the lack of support for representation of resources and implementation of business process in information systems. In addition, BPMN model simulations do not support concurrent use of the resources and it is not possible to graphically distinguish the independent processes and embedded processes [8], [17] and [22].

BPMN stands for a business process modeling language, which has become the default for modeling business process flow and Web services [4], [6], [7] and [21]. Web services are usually used for integrations of information systems or the automation of information processing activities. The activities, which cannot be automated or implemented as Web services in information systems, are excluded from such a business process model. Consequently, the real resources are irrelevant for such models, and resources are not represented. However, BPMN is used for modeling the real business processes with all process activities. In addition, such BPMN models try to simulate the tools based on BPMN, but in this case the simulation is insufficient. Model for business process simulation should include all activities of real business processes. Therefore, such a model needs more details of business process, e.g., duration activities, used resources, executed instances of the process, limitation of the resources or concurrent usage of resources. Therefore, BPMN needs an extension to support business process simulation.

The aspect of resources is critical in the business process simulation. Each of the resources is associated with an activity, which is indicated in the processes. Resources perform the work related to the following resources [4]. There are no business processes without resources. Business process needs to have described resources and workflow in order to be simulated. It is necessary to ensure that various activities use necessary resources or activities, which are carried out by the necessary resources (examples: personnel, worker, and equipment). Excessive activity automation and poorly planned distribution of work are critical moments of projects, which are based on a workflow [16]. Therefore, it is necessary to indicate resources for both: the business level and information (technical) level. BPMN 2.0 allows assigning resources, but does not allow for graphical representation of resources in a way that can be understood by non-experts [8], [16].

There are some other aspects that are necessary for business process simulation [19]. The simulation requires a more detailed business process analysis, investigation in the duration of activities and incoming workflow, which is not described in the standard business process model. It is necessary to investigate the duration of the activity and to explore a workflow. Activities of programmatically submitted simulation models need to be represented as processes with certain parameters, resources, attributes, and relationships. It must contain all relevant information about the activities because simulation is a representation of the mathematical calculations to a certain level. It requires the simulation engine and tool or software code, which can use this engine in order to simulate the model.

This paper analyzes the suitability of BPMN for modeling and simulating business processes with a particular emphasis on the resource aspect. This paper also analyzes the methods that allow proceeding the modeling of resources with BPMN based tools. It presents the evaluation of resource modeling in the business processes, highlighting the drawbacks of BPMN, when BPMN is used for business process modeling and simulation. Finally, the paper presents significant properties for resource modeling and proposes a method for resource modeling in BPMN models.

The structure of this paper is as follows: Section II is devoted to related work, Section III estimates the methods that allow modeling resources, and Section IV is a proposal of a method. 


\section{RELATED WORK}

Business process is a workflow lined up in logical order that creates a beneficial outcome for a recipient. Simulation execution and evaluation are known as business process management [6], [24]. Business process models are designed in order to represent and simulate the real process on the computer. Usually, the experts of the business domain area create business process models. These models are the starting point for software developers, who create these models by the original application system implementation [5], [17] and [24].

BPMN language is a graphical modeling notation widely used for modeling business processes in organizations [15], [11]. This notation is focused on process sequences [11]. A particular action or set of actions with certain resources are displayed graphically using an item called Swimlanes in the BPMN notation. This element presents the participants of the process. A participant may be a specific business entity or a general business rule. Resources are not necessarily allocated during the design in this notation. One of the major drawbacks of this notation is the lack of ability to model the resources [6], [8].

The author of the paper [21] suggests that the BPMN element called Swimlanes should be portrayed as the role and that each of these elements represents a certain role. However, while using such a method, business process model complicates a number of activities.

Other popular process modeling methods are IDEF0 and IDEF3. These languages originate from IDEF (Integration Definition). IDEF is a set of modeling languages in the field of systems and software engineering. IDEF0 was designed for creating a function model of a new or existing system of application domain. IDEF3 Process Description Capture Method is a method for creating a dynamic model of the system [3].

There are some approaches that are used for business process simulation. Montevechi et al. [10] proposed a technique for conceptual modeling called IDEF-SIM by adapting IDEF0 and IDEF3. In addition, IDEF-SIM uses symbols from IDEF0, IDEF3 and flow-chart, yet within a logic that reflects the simulation and is similar to the logic used in discrete event simulation. Jeong [9] proposes another approach, which is based on IDEF0 and IDEF3 as well. These models were used in the requirement collection phase. Then the authors proposed creating IDEF1X-based data model and this model became a knowledge base for simulation model development. Summarizing, IDEF based simulation approaches use IDEF models to capture requirements. After that, the authors propose creating a simulation model using another language and IDEF models for validation and verification of simulation model. However, the purpose of this paper is not the deep review of business process simulation based on IDEF, but the paper presents a review of approaches to modeling resources using BPMN extensions.

The paradigm of business process management systems is developed for business process modeling based on the workflow concept [1]. This conception becomes de-facto standard and usually consists of three perspectives:
- Perspective of control flow describes the logical order of a task. It represents an aspect of process control. There are six main groups of patterns that represent the perspective of control flow: basic control flow, advanced branching and synchronization, structural, multiple instances, state based, and cancellation [14], [22];

- Perspective of data flow describes the exchange of information between tasks. This perspective focuses on identifying and defining generic constructs for representation and handling data within process aware information systems. There are four groups of patterns that represent the perspective of data flow: data visibility, data interaction, data transfer, and data-based routing [23], [13].

- The perspective of resources describes the initiator of a task. This perspective focuses on the manner, in which work is distributed amongst and managed by the resources in a process-aware information system (PAIS). There are seven groups of patterns that represent the perspective of resources: creation, push, pull, detour, auto-start, visibility, and multiple resource [23], [12].

Business process modeling is only the first step in optimizing business processes. One of process model goals is to describe how business must, have, or can be made. Such an analysis helps to avoid the errors of the process design. Later on, these models are used in improvement programs of business processes. These models help to improve communication between business members and the developers of information systems, understand and transform the business and define situations and their consequences. Apart from that, the models help to evaluate the process changes before they are implemented, to identify potential costs, resource requirements, to identify and remove inefficient process areas and to provide changes in the market. A properly constructed business process models can help to improve business performance. A process model is a possible image of how the process will look like [11]. Business process models are usually static. Thus, in order to make important decisions and assess their impact, there is a need for simulation that shows how the individual objects behave. This is useful for testing, analysis or training sessions where the real world systems may reflect the models. Rarely, after a number of changes and assessment of their impact and real solutions, the cost can still be high for the best solutions of overall organizational success. The model usually reflects some aspects of this phenomenon. Many organizations try to use business process models to analyze their business processes in some states [2], [8].

The simulation is not sufficiently developed for the usage, as there is an important step in the business process management approach. The main idea of the simulation is that the model has to be executed several times repeatedly. This process is done to obtain more than one value, resulting in the range of values [20]. Usually the beginning of the simulation starts with filling business process model up with data. Once it is filled with data, the calculations would start. The simulation is rarely used in business decision-making; however, it is often 
applied to analyze the initial business process model. While many organizations try to use the simulation to analyze the changes in the business, only a few organizations use the simulation effectively. This situation occurs due to the fact that the development of simulation and supervision is a timeconsuming process. Often the simulation results may not correspond to the reality. Moreover, the organizations have a need to deal with issues that are relevant to the present time rather than abstract future problems [6], [18].

The business process models are often excluded from the simulation in the current modeling and simulation tools. Models and simulations are often being developed separately. Most of the simulation tools provide only the number of resources used. It is also common that organizations model resources incorrectly in the business process models and simulations. The problematic areas regarding a couple of the main resource modeling cases are identified below [18]:

- Some of the resources are involved in the several processes, such as human resources are often involved in multiple processes. However, business process simulation focuses only on a single process. For example, specific human resource is involved in ten different processes and each spends about $20 \%$ of their time. In most cases, it is not possible to simulate the situation with simulation tools. Therefore, simulation models consider that the resources are available all the time and have very little use. In this case, the simulation results are very inaccurate. Highly advanced simulation tools allow indicating that the resource is used at a given time. However, it is also the wrong abstraction for assigning resources in work processes in accordance with the priorities and workloads. In this case, the simulation results can also be inaccurate.

- The resources are involved in two or more instances of the ongoing (executing) process. This fact is important when resource carries out some activities. The resource is used at the same time in several activities of the same business process instance. Usually, business process simulation tools do not simulate such situations.

- When the resources do not work at a constant speed, e.g., human resources work at different speeds depending on the workload. Currently, there are no simulation tools that would allow modeling business process execution time, which is dependent on workload.

- Resources (for example, humans) decide themselves how to carry out the process.

- Some of the resources only work part-time. For example, human resources tend to work part of their time in a single process or to work in groups. Resources are not available or are available in most simulation tools. If the resources are available, they wait for work and they begin to work on the first occurrence of the process.

- When the process may change depending on the context. Most of the simulation tools consider that the processes are stable and do not change over time. If the flow of time is too long the resources may decide to skip certain activities or to use additional resources.
- Mentioned resource modeling problems show that the simulation results can be a significant deviation from the real world processes. The simulation model should have an appropriate level of details, and the addition of more information does not always solve the problem.

- Often, a business process has the limitation of resources, and, as a result, the concurrency may appear. A concurrency can be found in business, when two or more jobs (processes) are executed simultaneously, potentially interacting with each other when using the same resources [19].

Business process models and simulation resources are used when the system performs the entity. Resources include fixed equipment, mobile equipment, containers, personnel, supplies used and any other technical and material supplies that are needed to process an essence. The resources are divided into human and technical ones. They are also divided into quantitative, schedule availability, size, price, and cost management. Resource modeling describes employed or utilized resources in the business process. The resource modeling describes the usage of resources.

\section{Resource Modeling TeChNIQUes AND THEIR EVALUATION}

This section analyzes the resource modeling with BPMN tools and proposed extensions. There is a table that shows specific properties in the selected tool or extension (Table I).

TABLE I

TOOLS AND METHODS FOR SUPPORT OF RESOURCE MODELING

\begin{tabular}{|c|c|c|c|c|c|c|}
\hline $\begin{array}{l}\text { Tools for BP } \\
\text { modeling } \\
\text { and simulation }\end{array}$ & 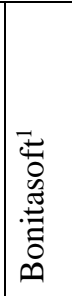 & 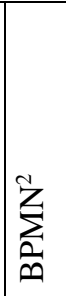 & 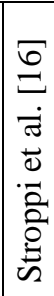 & $\begin{array}{l}\stackrel{n}{\tilde{n}} \\
\stackrel{n}{n}\end{array}$ & 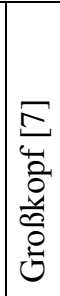 & 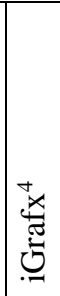 \\
\hline $\begin{array}{l}\text { Graphical } \\
\text { representation }\end{array}$ & - & $-1+$ & + & - & + & $-1+$ \\
\hline Duration parameters & + & - & - & + & - & + \\
\hline Priority & - & - & + & - & - & - \\
\hline Cost parameters & + & - & - & - & - & + \\
\hline Quantitative parameters & + & $-1+$ & + & + & - & + \\
\hline Roles & $-1+$ & + & + & + & + & $-1+$ \\
\hline Structure & - & - & + & - & - & - \\
\hline Availability parameters & - & - & - & $-1+$ & - & $-1+$ \\
\hline Concurrency & - & - & - & - & - & - \\
\hline
\end{tabular}

Table I shows a set of resource properties:

- Graphical representation - considering the proposed extension, tool has the ability to graphically display resources.

- Duration parameter of resources - considering the proposed extension, tool has the ability to specify the

\footnotetext{
www.bonitasoft.com

2 www.omg.org

${ }^{3}$ www.BPSim.org

4 www.igrafx.com/gl
} 
parameters that specify the time when the resource is available (range when it is available).

- Resource priority - it is used to indicate the resource priority that shows activities in which participation is important. This property also considers which activity is more important and which one should be performed first.

- Cost of resource usage - considering the proposed extension, tool has the ability to specify a parameter that indicates estimated resource utilization costs. The cost of the resource could be the price for usage or the price per time unit (for example, 10 Euro per hour).

- Roles - this resource can be assigned to one or several roles. One resource could have several roles.

- Structure [16] - refers to the characterization and classification of resources. The characterization describes different features of the resources, and the classification describes the association of resources with a concept. For example, classification can be a functional (roles) or organizational (organizational units).

- Quantity - considering the proposed extension, the tool has the ability to specify a certain quantity of a specific resource. The quantity is especially significant during simulation of process because almost all resources are in business. The fact that the resource is wasted has to be emphasized.

- Availability parameters - it considers the original state of the resource if the resource is available. It is important during the process of simulation. The activity that uses the resource should mark that the resource is unavailable. This parameter can be Boolean. However, the availability is not always important if the resources are unlimited.

- Concurrency - shows that the resource can be used concurrently. For example, the resource can be used concurrently of two or more ongoing business processes at the same time. This property is significant during the process simulation.

According to these properties, six extensions and tools have been revieved that are further discussed in this section.

BonitaSoft - is open source business process management software. It has three main components:

- Bonita Studio, which allows the modeling of business processes in BPMN standard;

- Bonita BPMN engine, which is written in the Java programming language;

- Bonita website - this tool has the ability to specify the time and quantity parameters. It has also the ability to assign a certain level or resource roles.

The simulation tool of BonitaSoft allows specifying resources for the business process. BonitaSoft model is based on BPMN. However, this tool cannot represent resources graphically in the process model or define the resource model separately from the business process. This tool provides standard forms to specify resources. BonitaSoft allows specifying the availability of resources. The tool allows specifying available quantity, target quantity, resource cost parameters such as cost unit, and cost per use or time cost (cost per minute, hour and day).
BPMN - this is a graphical notation intended to model business processes. This notation has four graphics categories. The first category consists of flow objects: activities, events, and gateways. The second category consists of connection objects: sequence flow, message flow, and association. The third category - swimlanes: a pool and a line. The last group is artefacts: data objects, groups, and annotations. BPMN allows defining business processes graphically. It also allows specifying roles using a pool and a line. These objects are used to specify resources graphically in some way. However, BPMN has the limitation: by using a pool and a line one cannot specify the resource quantity or it is difficult to represent many resources that are used in one task of business process. If one task uses more than one resource, then the pool and lines should be represented for all resources. Apart from that, some information resources can be presented using data objects that are input or output of a task.

Stroppi et al. [16] - is BPMN 2.0 extension for modeling of resources. This approach extends the BPMN meta-model, so that the comment element should be able to present not only comments, but also the resources and some information related to resources. This proposed method allows specifying the roles of resources, resource priority and even suggests graphical representation. This approach also proposes an extension for definition of resource structure. The resource structure is defined in a separated diagram. This extension suggests displaying resources using comment artefact in BPMN diagram. Further, this extension is used for process execution and for converting execution languages to other business processes like BPELAPeople. However, the method should be extended for usage in the simulation.

BPSim (Business Process Simulation) [25] - is proposed for the business process simulation specification. BPSim provides a framework or a standardized specification for capturing business process models while using BPMN or XPDL. BPSim defines the parameterization and interchange of process analysis data that allows structural and capacity analysis of process models. The BPSim framework distinguishes a resource parameter class. This class defines availability, quantity, selection and role parameters. In addition, it is distinguished by a cost parameter class. However, this class is not related to resource and the cost should also be defined for the use of resources. The selection parameter is a property for selecting the desired resource and it is similar to a priority parameter. However, this framework does not define how to represent resources in the business process model.

The method proposed by Großkopf [7] is an extension, which displays certain resource attributes. This approach particularly focuses on the role of resource definition. The comment element is converted to the element, which represents a specific number of parameters of resources. This extension offers a graphic representation and the way to assign the roles. However, these roles are task or activity performers. The approach does not deal with other resource types. The method does not define the quantity or cost of resources; it does not also define availability for the resource. 
iGrafx is a complete and easy to use process analysis and simulation tool that is specifically designed to help Six Sigma professionals to better understand and improve the process diagrams. This tool offers process design, analysis, and optimization and management capabilities. iGrafx allows modeling resources and assigning them to process activities. There is a worker tab, which allows manipulating the resources. However, the simulation with iGrafx does not use BPMN. Instead of BPMN iGrafx uses its own notation that is similar to BPMN. iGrafx notation represents resources by using swimlines and pools. It could be assigned by various parameters of resources, like time and cost. Availability parameter could be defined only as a percentage value and cannot be changed or influenced during simulation. The definition of resource roles is also limited, since resources cannot be assigned to different roles. Apart from that, the priority of resources cannot be defined.

\section{Proposed Method For Resource Modeling}

According to results (Table I), it is safe to state that there is not a suitable way to model resources and adequate graphical representation. Therefore, this article suggests a method, which attempts to collect advantages from each analyzed method and adapt these advantages to the reviewed approaches.

We propose creating a graphic element such as BPMN "comment". This new element should have new functionality that is designed to model resources. The element must have attribute, whose value can be selected from a drop-down list. Drop-down list contains names of the existing resources of the business process. Collapsed display represents only selected resource name (Fig. 1).

The expanded element would show all information that is related to a specific selected resource (Fig. 2).

Stroppi et al. [16] have proposed the visualization method, and this paper proposes expanding functionality, attributes, and parameters. Moreover, instead of having to use a comment element directly, we propose creating a duplicate of this element and modifying it to apply the new functionality. It is planned to implement the proposed method using a practical way in the Bonitasoft software. We have created an example of business process by adapting the method suggested (Fig. 3).

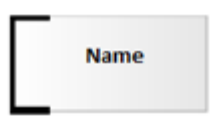

Fig. 1. Abstract graphic representation of a resource.

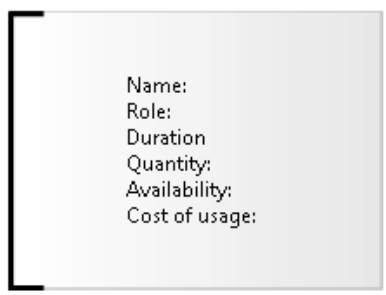

Fig. 2. Expanded element with resource information.

The proposed approach helps better understand the process that is designed for simulation. Process visualization is one of

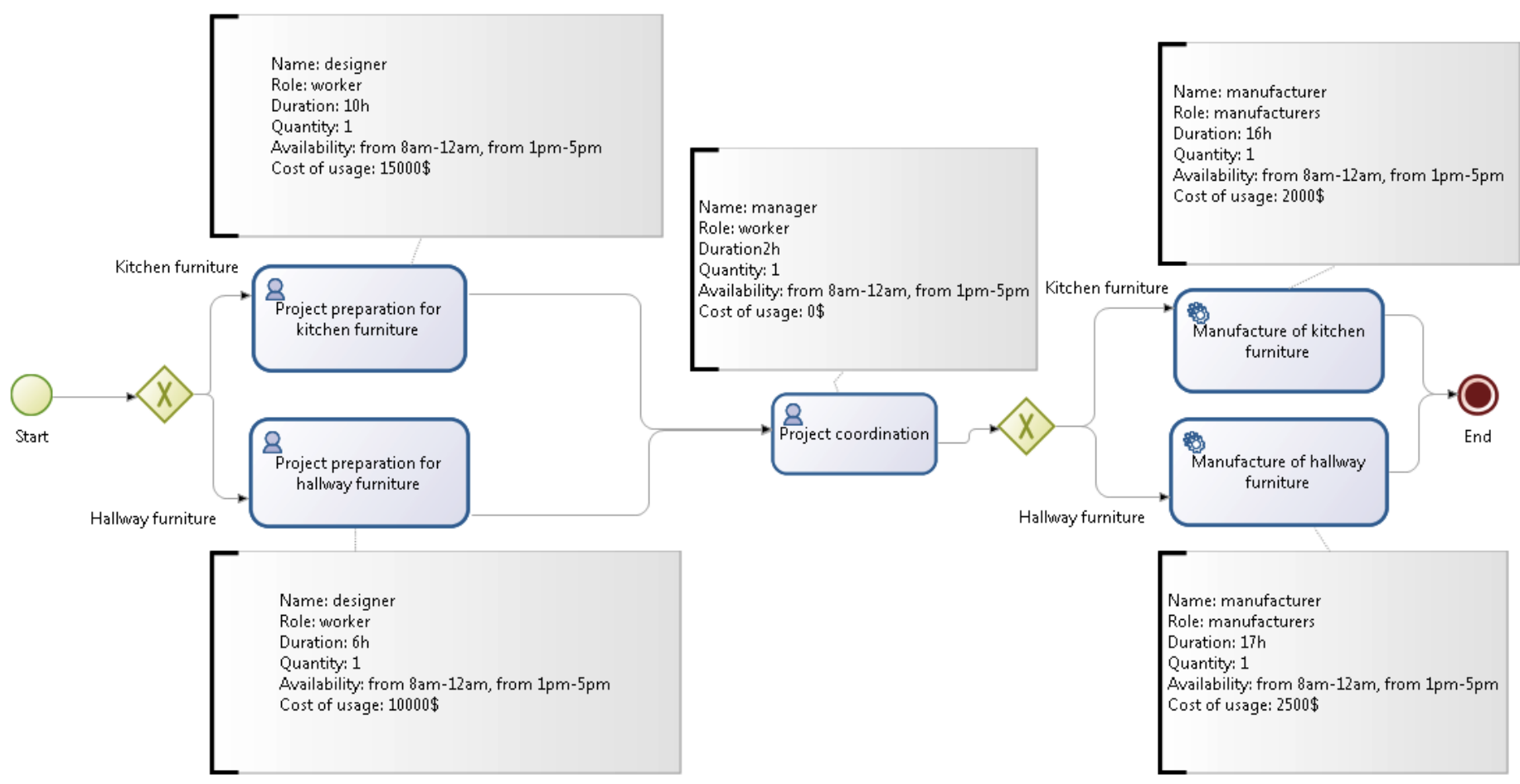

Fig. 3. Business process example with the proposed method (resource elements are expanded). 
the key factors in understanding the business process. This approach allows visualizing the process with resources and their parameters, such as cost quantity, priority and others. We propose two ways for visualization. The first way is only a resource name related to an activity without resource details (it is shown in Fig. 1). This way is more suitable for business people and it is not very overloaded. The second way is visualized resources with details (it is shown in Fig. 2 and Fig. 3). This way is most suitable for developers. However, the disadvantage of this method is that the model could be very complicated and difficult to understand.

We do not find an approach that could visualize all resource parameters. Despite that, there is not an approach that takes into account and could model all aspects of the resource. This approach could be implemented in business process simulation tools using a business process simulation metamodel of BPSim framework. This approach also takes into account the resource concurrency that is important when resources are limited.

\section{CONCLUSION AND FUTURE WORK}

BPMN language is not sufficiently adapted to model the resources. Resources are the important aspects of business process simulation. It can be checked only the workflow logic without aspect of resources using only BPMN model. The modeling of a resource aspect gives an opportunity to simulate the real world processes without performing the process in reality. In this way, there is a way to get the results and adapt them to the optimization and improvement of a real process.

The authors of the paper have selected and assessed methods and tools related to the BPMN modeling and simulation based on resource properties. It can be seen from the presented evaluations that there does not exist tool or method that allows one to model resources, provides the ability to specify particular settings and has a graphical notation.

The method proposed by Stroppi et al. [16] is one of the best ways from analyzed approaches to model resources in the graphical way. The method has been used as a foundation. The authors of this paper have proposed some changes that would broaden and give an opportunity to depict the resources with detailed information.

The proposed method has several interconnected advantages in comparison with existing methods and tools. The method offers to graphically display resources with essential parameters of resources like role of resource, availability, cost, duration, cost, quantity and concurrency. These improvements helps better understand the process that is designed for simulation.

Bonitasoft tool is an open source tool and is freely available to everyone. Apart from that, the source code is available. We have compiled the Bonitasoft source code and made the first changes in the source code. Further, we are planning the practical implementation of the proposed method by modifying the Bonita source code and developing a business process model for testing purposes.

\section{REFERENCES}

[1] M. Bajec and M. Krisper, "A methodology and tool support for managing business rules in organisations," Information Systems, vol. 30, no. 6, pp. 423-443, 2005. http://dx.doi.org/10.1016/j.is.2004.05.003

[2] M. Chinosi and A. Trombetta, "BPMN: An introduction to the standard," Computer Standards \& Interfaces, vol. 34, no. 1, pp. 124-134, 2012. http://dx.doi.org/10.1016/j.csi.2011.06.002

[3] C. Badica and C. Fox, "Hybrid IDEF0/IDEF3 modeling of business processes: Syntax, semantics and expressiveness," in RomanianAustrian Workshop on Computer-Aided Verification of Information Systems, 2004, pp. 20-22.

[4] R. M. Dijkman, M. Dumas, and C. Ouyang, "Semantics and analysis of business process models in BPMN," Information and Software Technology, vol. 50, no. 12, pp. 1281-1294, 2008.

http://dx.doi.org/10.1016/j.infsof.2008.02.006

[5] T. Dufresne and J. Martin, "Process modeling for e-business," Information Systems Department, George Mason University, 2003.

[6] L. J. Enstone and M. F. Clark, "BPMN and simulation," Technical report, Lanner Group Limited, 2006.

[7] A. Großkopf, "An extended resource information layer for BPMN," Hasso-Plattner-Institute for IT Systems Engineering, University of Potsdam, 2007.

[8] R. P. Jefferis, "Simulation of business processes," Widener University, 2001.

[9] K.-Y. Jeong, L. Wu, and J.-D. Hong, "IDEF method-based simulation model design and development framework," Journal of Industrial Engineering and Management, vol. 2, no. 2, pp. 337-359, 2009. http://dx.doi.org/10.3926/jiem.2009.v2n2.p337-359

[10] J. Montevechi, F. Leal, A. F. de Pinho, R. F. da Silva Costa, M. L. M. de Oliveira, A. L. F. da Silva, "Conceptual modeling in simulation projects by mean adapted IDEF: An application in a Brazilian tech company," in 2010 Winter Simulation Conference, 2010, pp. 1624-1635.

[11] M. Owen and J. Raj, "BPMN and business process management: Introduction to the new business process modeling standard," 2003.

[12] N. Russell, W. M. P. van der Aalst, A. H. M. ter Hofstede, and D. Edmond, "Workflow resource patterns: Identification, representation and tool support," in 17th International Conference on Advanced Information Systems Engineering, 2005, pp. 216-232. http://dx.doi.org/10.1007/11431855_16

[13] N. Russell, A. H. M. ter Hofstede, D. Edmond, and W. M. P. van der Aalst. "Workflow data patterns," in 24th International Conference on Conceptual Modeling, 2005, pp. 353-368.

[14] N. Russell, A. H. M. ter Hofstede, and N. Mulyar, "Workflow control-flow patterns: A revised view," Technical report BPM-06-22, 2006.

[15] N. Russell, W. M. P. van der Aalst, A. H. M. ter Hofstede, and P. Wohed, "On the suitability of UML 2.0 activity diagrams for business process modeling," in 3rd Asia-Pacific Conference on Conceptual Modeling, vol. 53, 2006. pp. 95-104.

[16] L. J. R. Stroppi, O. Chiotti, and P. D. Villarreal, "A BPMN 2.0 extension to define the resource perspective of business process models," in XIV Iberoamerican Conference on Software Engineering, 2011, pp. 25-38.

[17] N. Trcka, W. van der Aalst, and N. Sidorova, "Analyzing control-flow and data-flow in workflow processes in a unified way," Computer science report 08-31, 2008.

[18] W. van der Aalst, J. Nakatumba, A. Rozinat, and N. Russell, "Business process simulation: How to get it right," BPM Center report BPM-08-07, 2008.

[19] O. Vasilecas, A. Smaižys, and A. Rima, "Business process modeling and simulation: Hybrid method for concurrency aspect modeling," Baltic Journal of Modern Computing, vol. 1, no. 3-4, pp. 228-243, 2013.

[20] V. B. Vukšić, V. C. Ceric, and V. Hlupic, "Criteria for the evaluation of business process simulation tools," Interdisciplinary Journal of Information, Knowledge \& Management, vol. 2, pp. 73-88, 2007.

[21] M. Weske, "Evolution of enterprise systems architectures," in Business Process Management. Springer, 2012, pp. 25-69.

[22] P. Wohed, W. M. P. van der Aalst, M. Dumas, A. H. M. ter Hofstede, and N. Russell, "On the suitability of BPMN for business process modeling," in 4th International Conference on Business Process Management, 2006, pp. 161-176. http://dx.doi.org/10.1007/11841760 12

[23] P. Wohed, M. Dumas, A. H. M. Ter Hofstede, and N. Russell, "Patternbased analysis of BPMN - An extensive evaluation of the control-flow, the data and the resource perspectives," 2005. 
[24] M. zur Muehlen and M. Indulska, "Modeling languages for business processes and business rules: A representational analysis," Information Systems, vol. 35, no. 4, pp. 379-390, 2010. http://dx.doi.org/10.1016/j.is.2009.02.006

[25] Workflow Management Coalition, "Business process simulation specification," document number WFMC-BPSWG-2012-1, version 1.0 2013. [Online]. Available: http://www.bpsim.org/specifications/ 1.0/WFMC-BPSWG-2012-01.pdf.

Olegas Vasilecas, prof. (hp) dr., is full time professor at the Information Systems Department, principal researcher and head of Information Systems Research Laboratory in Vilnius Gediminas Technical University. He is author and co-author of more than 370 research papers and 5 books in the field of information systems development. He delivered lectures in 7 European universities including London, Barcelona, Athens and Ljubljana. O. Vasilecas carried out an apprenticeship in Germany, Holland, China, and last time in Latvia and Slovenia universities. He supervised 11 successfully defended doctoral theses and now is supervising 4 more doctoral students. He was leader of number of international and local projects. Last time he leaded
"Business Rules Solutions for Information Systems Development (VeTIS)" project carried out under High Technology Development Program of Lithuania. Research interests include knowledge based information systems, knowledge represented by business rules and ontology, knowledge extraction from information systems databases, dynamic business processes, information systems modernisation.

E-mail: olegas.vasilecas@vgtu.lt

Audrius Rima is a Doctoral Student at Vilnius Gediminas Technical University. He received the MSc degree in Informatics from Klaipeda University in 2007. His research areas are as follows: business process modeling and simulation.

E-mail: audrius.rima@dok.vgtu.lt

Evaldas Laureckas is a Student of Klaipeda University. Currently, he has been studying at the master study program. He received a Bachelor degree in Informatics from Klaipeda University in 2013. He also works as a Software Systems Engineer at the JSC "Omega Technology".

E-mail: evaldas.laureckas@gmail.com 